University of Wollongong

Research Online

Faculty of Engineering and Information

Faculty of Engineering and Information

Sciences - Papers: Part B

Sciences

2018

\title{
Tunable smart digital structure (SDS) to modularly assemble soft actuators with layered adhesive bonding
}

Hu Jin

University of Science and Technology of China

Erbao Dong

University of Science and Technology of China

Min Xu

University of Science and Technology of China

Qirong Xia

University of Science and Technology of China

Shuai Liu

University of Science and Technology of China

See next page for additional authors

Follow this and additional works at: https://ro.uow.edu.au/eispapers1

Part of the Engineering Commons, and the Science and Technology Studies Commons

Research Online is the open access institutional repository for the University of Wollongong. For further information contact the UOW Library: research-pubs@uow.edu.au 


\title{
Tunable smart digital structure (SDS) to modularly assemble soft actuators with layered adhesive bonding
}

\author{
Abstract \\ Many shape memory alloy (SMA)-based soft actuators have specific composite structures and \\ manufacture processes, and are therefore unique. However, these exclusive characteristics limit their \\ capabilities and applications, so in this article a soft and smart digital structure (SDS) is proposed that \\ acts like a modular unit to assemble soft actuators by a layered adhesive bonding process. The SDS is a \\ fully soft structure that encapsulates a digital skeleton consisting of four groups of parallel and \\ independently actuated SMA wires capable of outputting a four-channel tunable force. The layered \\ adhesive bonding process modularly bonds several SDSs with an elastic backbone to fabricate a layered \\ soft actuator where the elastic backbone is used to recover the SDSs in a cooling process using the SMA \\ wires. Two kinds of SDS-based soft actuators were modularly assembled, an actuator, SDS-I, with a two- \\ dimensional reciprocal motion, and an actuator, SDS-II, capable of bi-directional reciprocal motion. The \\ thermodynamics and phase transformation modeling of the SDS-based actuator were analyzed. Several \\ extensional soft actuators were also assembled by bonding the SDS with an anomalous elastic backbone \\ or modularly assembling the SDS-Is and SDS-IIs. These modularly assembled soft actuators delivered \\ more output channels and a complicated motion, e.g., an actinomorphic soft actuator with four SDS-Is \\ jumps in a series of hierarchical heights and directional movement by tuning the input channels of the \\ SDSs. This result showed that the SDS can modularly assemble multifarious soft actuators with diverse \\ capabilities, steerability and tunable outputs.

\section{Disciplines} \\ Engineering | Science and Technology Studies

\section{Publication Details} \\ Jin, H., Dong, E., Xu, M., Xia, Q., Liu, S., Li, W. \& Yang, J. (2018). Tunable smart digital structure (SDS) to \\ modularly assemble soft actuators with layered adhesive bonding. Smart Materials and Structures, 27 (1), \\ 015012-1-015012-12.
}

\section{Authors}

Hu Jin, Erbao Dong, Min Xu, Qirong Xia, Shuai Liu, Weihua Li, and Jie Yang 


\title{
Tunable Smart Digital Structure (SDS) to Modularly
}

\section{Assemble Soft Actuators with Layered Adhesive Bonding}

\author{
Hu Jin ${ }^{1}$, Erbao Dong ${ }^{1 *}$, Min Xu ${ }^{1}$, Qirong Xia ${ }^{1}$, Shuai Liu ${ }^{1}$, Weihua $\mathrm{Li}^{2}$, Jie Yang ${ }^{1}$ \\ ${ }^{1}$ Department of Precision Machinery and Precision Instrumentation, School of \\ Engineering Science, University of Science and Technology of China, Hefei, Anhui, \\ 230026, P.R.China \\ ${ }^{2}$ School of Mechanical, Materials and Mechatronic Engineering, University of \\ Wollongong, Wollongong, NSW 2522, Australia
}

\begin{abstract}
Many shape memory alloy (SMA)-based soft actuators have specific composite structures and manufacture processes, and are therefore unique. However, these exclusive characteristics limit their capabilities and applications, so in this article a soft and smart digital structure (SDS) is proposed to act like a modular unit to assemble soft actuators by a layered adhesive bonding process. The SDS was a fully soft structure that encapsulated a digital skeleton consisting of four groups of parallel and independently actuated SMA wires capable of outputting a 4-channel tunable force. The layered adhesive bonding process modularly bonds several SDSs with an elastic backbone to fabricate a layered soft actuator where the elastic backbone is used to recover the SDSs in a cooling process by the SMA wires. Two kinds of SDS-based soft actuators have been modularly assembled, an actuator SDS-I with a two-dimensional reciprocal motion, and an actuator SDS-II capable of bi-directional reciprocal motion. Thermodynamics and phase transformation modeling of SDS-based actuator were analysed. Several extensional soft actuators have also been assembled by bonding the SDS with anomalous elastic backbone or modularly assembling the SDS-Is and SDS-IIs. These modularly assembled soft actuators deliver more output channels and a complicated motion, e.g., an actinomorphic soft actuator with four SDS-Is jumps in a series of hierarchical heights and directional movement by tuning the input channels of the SDSs. This result shows that the SDS can now modularly assemble multifarious soft actuators with diverse capabilities, steerability and tunable outputs.
\end{abstract}

Keywords: Soft Actuator; Smart and Digital Structure (SDS); Shape Memory Alloy (SMA) wires; Layered Adhesive Bonding; Tunable

\section{Introduction}

Since the notion of 'robot' was proposed, robots have established a close relationship with human beings in industrial manufacture, in homes, and in entertainment. Using hard materials, electromagnetic components and precise sensors, man has manufactured many hard robotic systems with great precision, which are capable of high loads and are configurable. However, hard robots can only work 
efficiently in a conditional environment without disturbances or unpredictable situations, and unlike human beings, with a hard body. Biological organisms [1] have survived for hundreds and thousands of years, they are highly adaptive, robust, and agile in an unstructured environment. Some jumping robots [2-4] performed jumping movement with high robustness properties. Researchers have transferred the structure and control principles of biological systems to the design of soft robots to develop novel technical solutions [5-7]. With soft bodies, soft robots [8] can self-adapt, deform, and behave autonomously, just like objects that are touched, and therefore they cannot harm humans or the environment, just like real animals. For example, an arm-like actuator [9] inspired by octopus arm and a soft gripper [10] with three arms can safely grasp objects.

SMAs refers to alloys which are stretchable and self-sensing, possess high energy density and provide a clean and noiseless operation, and are now widely used in the in manufacture of soft actuators [11-12] and robots [13-14]. However, the notoriously non-linear behavior of SMA limits the precision and controllability of SMA-based actuators. Studies of SMA in modelling and control have attracted the attention of many researchers for decades, most of which has focused on describing and predicting the thermodynamic process of SMA by building many constitutive models [15-17] whose parameters are determined mainly by experimental data. Some works [18-21] achieved force and position control of SMA-based actuators usually by taking advantage of external sensors and additional control mechanisms. As the strain-resistance of SMA is roughly linear [22], its repeatability encourages the use of its resistance as a feedback sensor [23]. Based on feedback on SMA resistance, researchers have accomplished position control using neural networks [24], and an accuracy control in an antagonistic SMA-based mechanism [25] with a duty input and proper pre-strain in SMA. Moreover, researchers also derived some Laguerre models based on experimental results to predict and control the displacement of a linear [26] or antagonistic actuators [27]. It is easy to find that the currently models or methods to control SMA are based on experimental results, antagonistic or spring-biased SMA-based actuators, additional control elements and limited input.

SMA wires are a subclass of SMAs, and behave very much like superimposed functions of tendons and muscles that can easily be embedded into soft materials to fabricate as composite structures [28, 29] with great compatibility, flexibility, and adaptability. Normally, SMA wires-based soft actuators fabricate the SMA wires with an elastic structure which will recover the SMA wires to their original positions in the cooling process. For example, a smart soft structure [30] capable of twisting and bending was made from SMA wires and a tube, as an elastic structure. In our previous work, we designed a soft and smart modular structure (SMS) [31] consisting of several SMA wires, a PVC plate and PDMS. The SMS was manufactured by a molding and a layered casting technology from molds made by a 3D printer. Similarly, many works [9, 30] have used soft lithography to manufacture the composite structures into which SMA wires with elastic structures are integrated. The other interesting technology used in wafer-scale or micro SMA-based actuators is adhesive 
bonding [32], Au-Si eutectic bonding [33, 34] and electroplate bonding [35] processes. Compared to soft actuators using soft lithography, SMA-based actuators have thin, compatible structures based on these bonding methods.

Although there are many SMA-based soft actuators or robots, most of them apply the designation, fabrication, and locomotion principles of robots rather than the precision control of systems. This is reasonable because soft robotics has only become popular over the past ten years, and there are too many problems still existing in bionics, actuations and structures, etc. Although soft and deformable bodies reduce the need for precision modelling and control of soft actuators and robots, some have tried to precisely control soft actuators [31]; for example, a self-sensing model [36] was used in a muscle-like actuation to achieve angular control. Those preliminary works explore the possibility of precisely controlling SMA-based soft structures, but angular control and bending range control will only work effectively under a no load condition. The results apparently show that current models or methods for SMA-based actuators are impractical because they cannot satisfy the engineering application of SMA which involves a complicated load, unfinished phase transformation, and a dynamic response.

Based on 3D printer technology and soft lithography, the manufacture of soft robots is fast and extensible. With different molds, a soft pneumatic structure [37] can be fabricated as a three dimensional tentacle, a starfish-shaped gripper, and a quadruped crawling robot. However, this manufacturing process is irreversible and each kind of robotic system must have a unique design and set of molds. A modular assembly [38] on the other hand, refers to a manufacturing process reconfigurable robot systems based on modules are made. Harvard proposed a magnetic assembly method [39] to assemble soft robots in a series of modules with diverse morphologies. Based on the SMS [31], several kinds of robots were assembled and an actinomorphic soft robot in modules could crawl on the ground, swim underwater, and grasp fragile objects. These proved that the modular assembly process improved the compatibility, manufacturing efficiency, and capability of soft robots. To achieve progressive or multiple output, researchers have conducted some structural improvement in actuators. Rodrigue $\mathrm{H}$ et al applied two independently sets of SMA wires to achieve bending and twist motion of a SMA-based composite structure [29]. By considering the overall difficulty of modelling SMA and soft deformable structures, a novel concept has been proposed to achieve a tunable force output of soft actuators based on a digital structural design, an innovative structural fabrication and a modular assembly.

The objective of this paper is to assemble multifarious soft actuators with capabilities of tunable output and steerability based on digital structures and modular assembly method. In ours study, two types of modules are used to be soft actuator modules: one is a smart digital structure (SDS), and the others are SDS-based soft actuators. We first designed and fabricated the SDS, which consists of four groups of SMA wires, each of which can be actuated independently. This means the SDS can achieve a four-channel force output by heating a corresponding number of groups of SMA wires, after which the SDS can be used to modularly fabricate various soft and 
stretchable actuators with different working manners and dimensions by bonding with an elastic backbone using a layered adhesive bonding technology. Besides, the SDS-based actuators can act as modules to further assemble soft robots with multiple modes. These SDSs and SDS-based actuators can be fabricated in a thin structure using the layered adhesive bonding process, so they are highly compatible and reconfigurable. The main contributions of this paper includes three aspects: (1) proposing a layered adhesive bonding technology to fabricate soft actuators; (2) using digital structures that are SDSs and modular assemble method to assemble soft actuators with steerable properties and multiple modes and (3) particularly, an actinomorphic soft actuator capable of steerable jumping motion.

\section{Design, Fabrication and Method}

\subsection{Design and fabrication of the SDS}

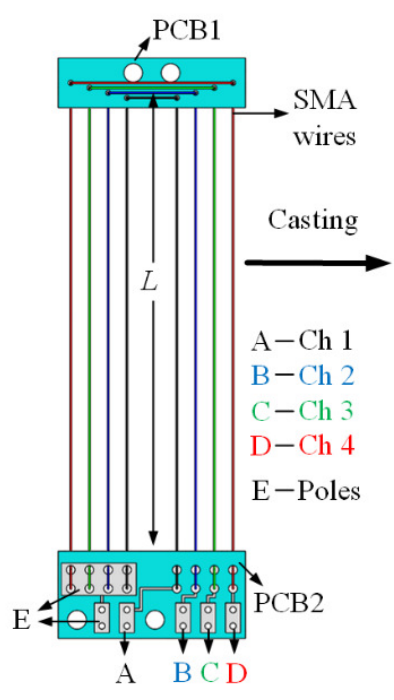

(a)

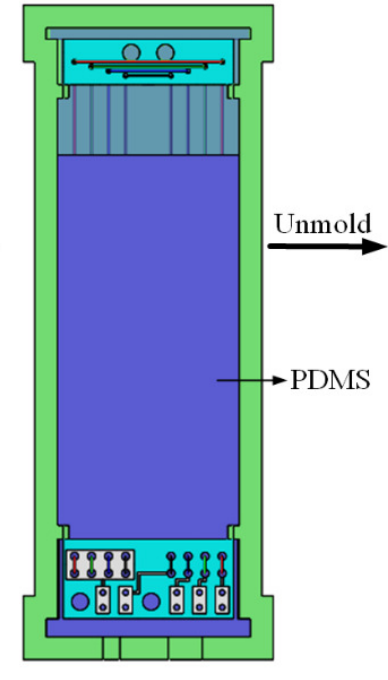

(b)

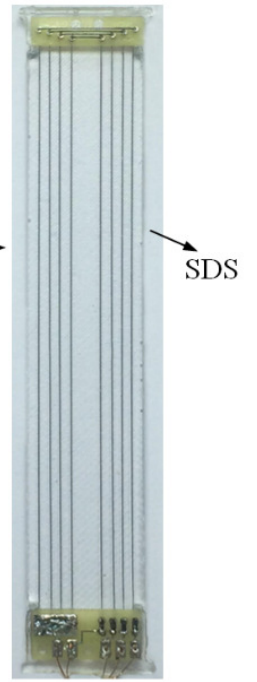

(c)

FIG. 1. Design and fabrication of the SDS. a) Digital skeleton of the SDS, b) fabrication of the SDS and c) the unmolded SDS.

The SDS consists of a digital skeleton and a soft body made from polydimethylsiloxane (PDMS). Casting technology was used to fabricate the SDS by casting the PDMS into a mold with the digital skeleton so that the PDMS will totally encapsulate the digital skeleton. The SDS is waterproof and is also a thin (the thinnest SDS is about $1.2 \mathrm{~mm}$ ) light structure which can be easily bonded or assemble with other structures. As shown in Figure 1a, the digital skeleton consists of two fixed plates and four sets of SMA wires with respect to four channels Ch1, Ch2, Ch3 and Ch4, respectively. All the channels have one shared poles E. Then, the casting technology was employed to fabricate the SDS based on mold casting process whose mold was produced by 3D technology as shown in Figure 1B. After solidification, as shown in Figure 1C, the SDS is transparent.

The SMA wires used in this paper are produced by Dynalloy Incorporation (diameters of $0.1 \mathrm{~mm}$ and $0.15 \mathrm{~mm}$, and transformation temperature $90{ }^{\circ} \mathrm{C}$ ). 
Polydimethylsiloxane (PDMS) was employed as the soft underlying material of the SDS and printed circuit boards (PCBs) to be the fix plates. The effective length of the SDS is $80 \mathrm{~mm}$, as $L$ shown in Figure 1a. The totally length and width of the SDS are $93 \mathrm{~mm}$ and $16 \mathrm{~mm}$, respectively. The height of the SDS is variable and can be regulated by redesigning the molds. In this paper, all the SDSs applied a height of 2.4 $\mathrm{mm}$ with a weight of $3.8 \mathrm{~g}$.

Layered Adhesive Bonding Technology to Assemble Soft Actuators

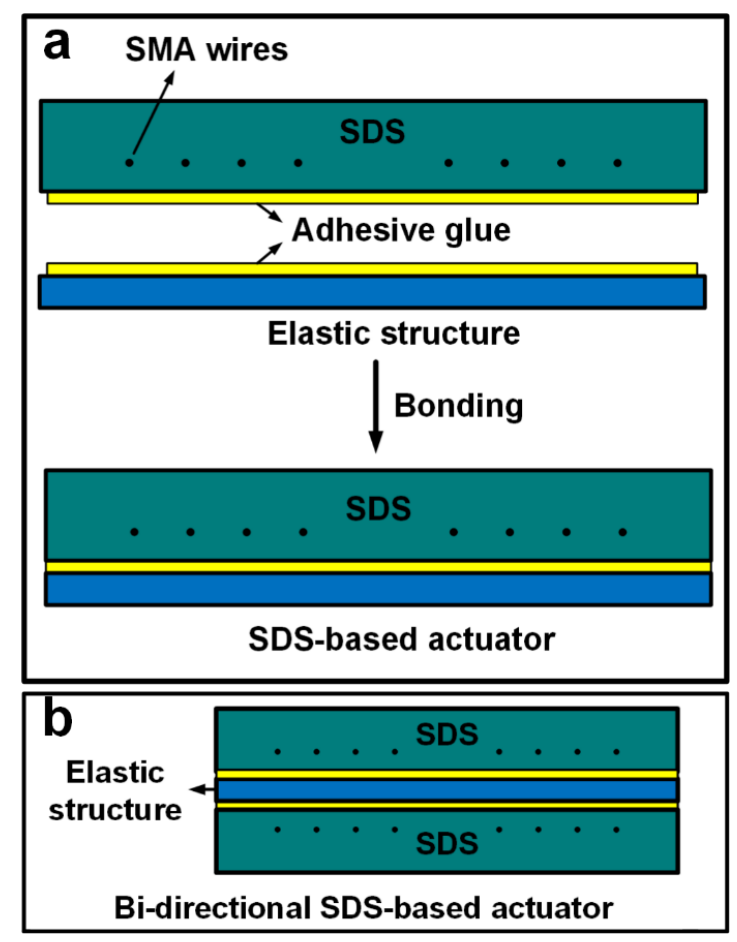

FIG. 2. a) Manufacture of SDS-based actuator by layered adhesive bonding based on SDS, b) bi-directional (SDS-II) actuator by assembling the SDSs using the adhesive bonding technology.

The technique for layered adhesive bonding is a fabrication process where adhesive glue (HS862 and HS880A, made by ANYBOND Inc.) is used to bond the SDSs with an elastic backbone to assemble the SDS-based actuator. This SDS-based actuator has a layered structure in which the elastic backbone is used to recover the SDSs while the SMA wires are cooling. Since SDSs are thin and soft, they are arranged on the elastic backbone with a slick and flat adhesive surface. Obviously, the architecture of the elastic backbone will decide the shape and function of the SDS-based actuator. Figure 2a shows how the SDS-based actuator is made by using the layered adhesive bonding process by bonding the SDS with a thin, flat, and slick laminated elastic backbone. This morphological SDS-based actuator is named as SDS-I, and it is capable of planar reciprocal motion where the actuator bends to the SDS side during the heating process and returns to its original position as it cools. There are many choices for the laminated elastic backbone; for example, a polyvinyl chloride (PVC) plate, a spring steel plate, and an acrylonitrile butadiene styrene (ABS) plate, etc. A $0.1 \mathrm{~mm}$ thick spring steel plate was used to fabricate the SDS-I in the 
following parts. A bi-directional SDS-based actuator called SDS-II, which is capable of bi-directional reciprocal motion, has been fabricated by bonding two SDSs onto the two sides of a laminated plate, as shown in Figure 2b.

\subsection{Tunable Method}

SMA wires have a complicated relationship and its temperature, strain, and stress are non-linear. In our work, the temperature-stress of the SMA wires was focused for two reasons: First, the flexible structures of an SMA-based soft actuators reduce the need for displacement control; and second, the controllable or tunable force output makes the SMA-based soft actuators have multiple or hierarchy performances which may be qualified in complicated tasks that barely exist in present day SMA-based soft actuators. As Figure 3a shows, a test platform has been designed to explore the tunable method of SDS. A force sensor (made by Zhongnuo Incorporation, model: ZNLBM-20N, precision $0.005 \mathrm{~N}$ ) connected linearly to the SDS and the signal of the force sensor was captured by an acquisition system (made by Advantech Incorporation, model: PCL-1716, resolution 16 bits). The sampling data was handled based on the DAQNavi software (made by Advantech Incorporation). Two series of experiments were carried out at heating currents of $0.4 \mathrm{~A}$ and $0.47 \mathrm{~A}$, with the same heating times of $2 \mathrm{~s}$ (open-loop control strategy) in all the experiments in this section.

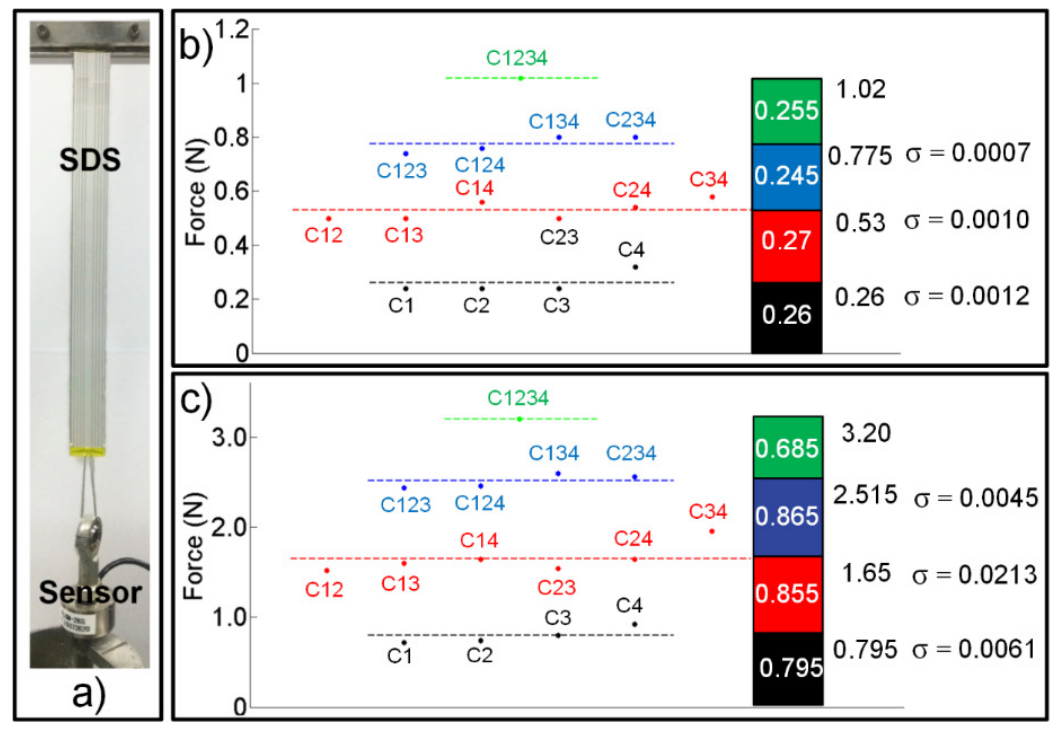

FIG. 3. Tunable method of the SDS. a) Experimental platform to measure the force of the SDS, b) and c) are the forces in four channels (totally fifteen combinations) under heating current of $0.4 \mathrm{~A}$ and $0.47 \mathrm{~A}$, respectively.

The digital arrangement of SMA wires gives the SDS four independent circuits. The combination with $n$ circuits was defined as the $n$-channel, which means there are fifteen combinations with one from 4-channel, six from 2-channel, and four from both 1-channel and 3-channel. The subscript of the 'Cxy' represents the combination with conducting circuits of 'Chx' and 'Chy'. Figure $3 \mathrm{~b}$ and $3 \mathrm{c}$ shows the results of tunable tests of SDS under heating currents of $0.4 \mathrm{~A}$ and $0.47 \mathrm{~A}$, respectively. At the low current input, the average forces of four kinds of channels are $0.26 \mathrm{~N}, 0.53 \mathrm{~N}, 0.775 \mathrm{~N}$ 
and $1.02 \mathrm{~N}$, respectively. At the current of $0.47 \mathrm{~A}$, the average forces of four kinds of channels are $0.795 \mathrm{~N}, 1.65 \mathrm{~N}, 2.515 \mathrm{~N}$ and $3.20 \mathrm{~N}$, respectively. These forces are larger in the higher heating current. Most importantly, both of the average forces in the two series of tests showed apparently hierarchical outputs of the four kinds of channels. Under the two current inputs, the mean square deviations of the output forces in all the combination were small enough, which proves the SDS in all four kinds of channels have steady outputs. Therefore, we can make a conclusion that the SDS can have four channelised, steady and tunable outputs.

\section{Modeling}

\subsection{Thermodynamics analysis}

To analyse the thermal transfer and heat accumulation of SDS-based actuators, a thermodynamic model was built based on COMSOL Multiphysics platform, as shown in Figure 4a. Based on the cross section area A-A, it is intuitively to observe the temperature field of the SDS-based actuator. The temperature field also shows the structural property of the SDS-I, where the temperature in the middle of the SDS-I is lower than its adjacent areas due to the arrangement of the SMA wires.
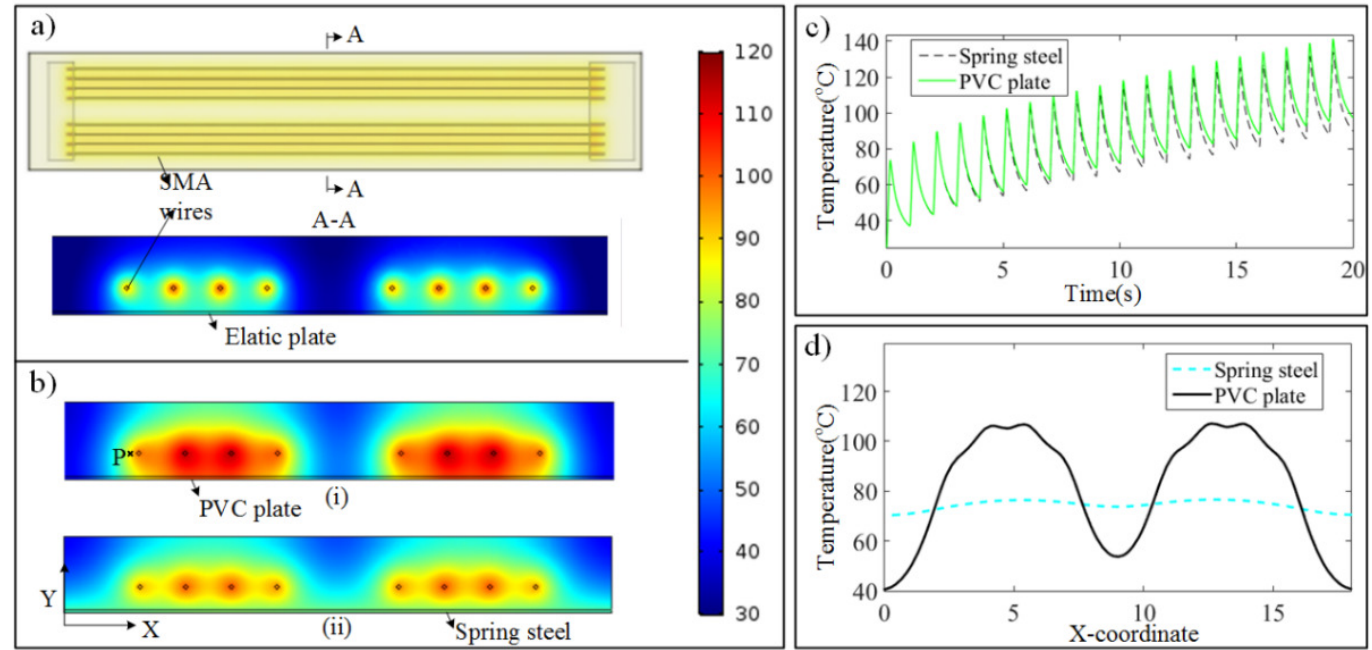

FIG.4. Thermodynamics of SDS-based actuator.

SDS-based actuators are composite structures which are composed of SDSs and elastic plates (detail in the next section). As a component, the elastic plate surely affect the heat transformation of actuators. A comparison was conducted between simulations in which two SDS-based actuators used a polyvinyl chloride (PVC) plate and a spring steel plate, respectively. In the simulations, the heating frequency is $1 \mathrm{~Hz}$ with a constant heating time of $145 \mathrm{~ms}$. Figure $4 \mathrm{~b}$ shows the temperature fields of two actuators after 20 cycles. Both of them presented heat accumulation phenomena in which the temperature of PDMS around the SMA wires was higher than those are far from the SMA wires. It is easy to find that the actuator with the PVC plate has a serious heat accumulation than the one with the spring steel.

To further illustrate the difference between the above two actuators, the 
temperatures of point $\mathrm{P}$ are presented in Figure 4c. The point $\mathrm{P}$ belongs to the structure of PDMS but is close to the SMA wires, as shown in Figure 4b (i). According to the Figure $4 \mathrm{c}$, the temperatures of point $\mathrm{P}$ presented fluctuation but increase trend. The fluctuation is identify to the heating and cooling processes of the SMA wires, and the increase trend is resulted by the heat accumulation. After 20 cycles, the temperature of the actuator with the spring steel is lower than the one with PVC plate. Figure $4 d$ shows the surface temperatures of the elastic plate whose coordination is shown in Figure $4 \mathrm{~b}$ (ii). The actuator with the spring steel presents a homologous temperature distribution than the one with the PVC plate. As such distribution means lower balance temperature in the structure around the SMA wires and better heat dispersion, it can be illustrate that the spring steel can improve the thermal performance of SDS-based actuators comparing to the previous composite actuator [26] with a PVC plate.

\subsection{Phase transformation modeling of SDS-based actuator}

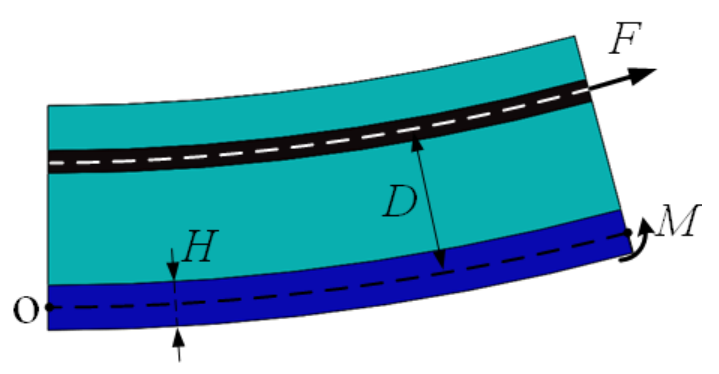

FIG.5. Schematics of the SDS-I actuator.

This part introduces the phase transformation modeling of the soft actuator SDS-I, which will be detail presented in the following parts. To simply the force analysis, three hypotheses were conducted: (1) the bending curvatures of the elastic plate of two actuators are constant, (2) the moment of the PDMS can be ignored as its Young's modulus is far smaller than the elastic plate and (3) the angular moment of the SDS-I is equal to zero.

To the soft actuator SDS-I, the stress of the SMA wires relate to the curvature of the elastic plate:

$$
\varepsilon_{0}-\varepsilon=\kappa D
$$

where $\varepsilon_{0}$ is the initial strain of the SMA wires, and $\varepsilon$ is the strain of the SMA wires. $\kappa$ is the curvature of the elastic plate and $D$ is the distance between the SMA wires and the elastic plate. The bending moment of the elastic plate is:

$$
M_{\mathrm{e}}=\int_{-H / 2}^{H / 2} E_{e} \kappa b y^{2} d y=E_{e} \kappa H^{3} b / 12
$$

where $E_{e}$ is Young's modulus of the elastic plate, $b$ and $H$ respectively are the width and thickness of the elastic plate. As shown in Figure 5, the resultant moment of the 
SDS-I is 0:

$$
F D=M_{\mathrm{e}}+M_{w}
$$

where $F$ is the force of the SMA wires, $M_{w}$ is the angular moment of the SDS-I. According to the hypotheses, $M_{w}$ is equal to zero. Therefore, the stress of the SMA wires can be derived as:

$$
\sigma=\frac{E_{e} H^{3} b\left(\varepsilon_{0}-\varepsilon\right)}{12 D^{2} A}=\Lambda\left(\varepsilon_{0}-\varepsilon\right)
$$

where $\Lambda$ is stress factor of the SMA wires and $\Lambda=\frac{E_{e} H^{3} b}{12 D^{2} A}, A$ is the sectional area of the SMA wires.

The constitutive model of the SMA wires could be expressed as below:

$$
\dot{\sigma}=E(\xi)\left(\dot{\varepsilon}-\varepsilon_{0} \dot{\xi}\right)
$$

where $E(\xi)$ is the Young's modulus of the SMA wires:

$$
E(\xi)=E_{A}+(1-\xi)\left(E_{M}-E_{A}\right)
$$

Where $E_{A}$ and $E_{M}$ are Young's modulus of the SMA wires in the austenite and martensite phases, respectively. $\xi$ is the martensite fraction of the SMA wires and is expressed as following in the austenite phase transformation process:

$$
\xi= \begin{cases}\frac{\xi_{M}}{2} \cos \left[a_{A}\left(T-A_{S}\right)+b_{A} \sigma\right]+\frac{\xi_{M}}{2} & \mathrm{M} \rightarrow \mathrm{A} \\ \frac{1-\xi_{A}}{2} \cos \left[a_{M}\left(T-M_{f}\right)+b_{M} \sigma\right]+\frac{1+\xi_{A}}{2} & \mathrm{~A} \rightarrow \mathrm{M}\end{cases}
$$

where $a_{A}=\pi /\left(A_{f}-A_{s}\right), b_{A}=-a_{A} / C_{A}, a_{M}=\pi /\left(M_{s}-M_{f}\right), b_{M}=-a_{M} / C_{M}$, and $C_{A}$, and $C_{M}$ are the effect of stress on the austenite and martensite temperatures of the SMA wires, respectively. $\xi_{M}$ and $\xi_{A}$ respectively are Initial martensite fraction and martensite fraction in austenite finishing.

Under a heating current of $I$, the thermal model of the SMA wires can be express as below:

$$
\rho c V \dot{T}=I^{2} R-\left(T-T_{0}\right) / R_{\lambda}+\rho V h \dot{\xi}
$$

where $h$ is the latent heat of transformation of the SMA wires. $\rho$ and $c$ respectively are the density and specific heat capacity of the SMA wires. $V$ and $R$ are the volume and resistance of the SMA wires, respectively. $T_{0}$ is the environmental temperature, and $R_{\lambda}$ 
is the thermal resistance of the PDMS:

$$
R_{\lambda}=\frac{1}{2 \pi \lambda l_{S M A}} \ln \left(\left(d+d_{p d m s}\right) / d\right)
$$

where $\lambda$ is the coefficient for the thermal conductivity of the PDMS, $l_{S M A}$ is the length of the SMA wires and $d_{p d m s}$ is the radius of the PDMS encasing the SMA wires.

According to equations of (4) - (9), the phase transformation model of the SDS-I was derived as shown in following:

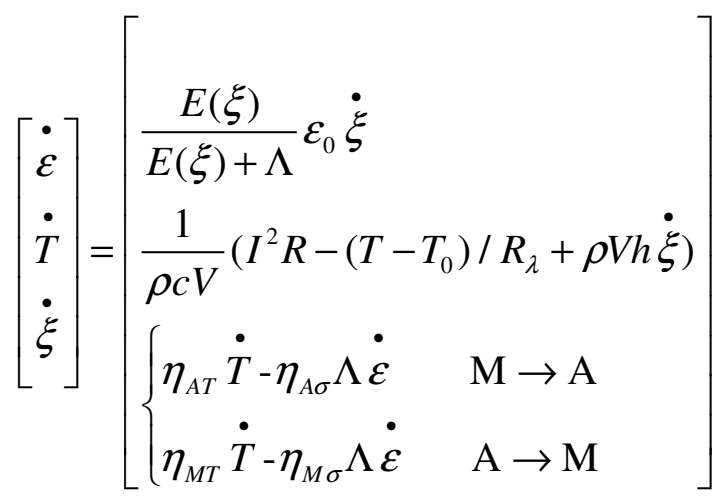

where $\quad \eta_{A T}=-\frac{\xi_{M} a_{A}}{2} \sin \left[a_{A}\left(T-A_{S}\right)+b_{A} \sigma\right], \quad \eta_{A \sigma}=-\frac{\xi_{M} b_{A}}{2} \sin \left[a_{A}\left(T-A_{S}\right)+b_{A} \sigma\right]$ $\eta_{M T}=-\frac{\left(1-\xi_{A}\right) a_{M}}{2} \sin \left[a_{M}\left(T-M_{f}\right)+b_{M} \sigma\right], \eta_{M \sigma}=-\frac{\left(1-\xi_{A}\right) b_{M}}{2} \sin \left[a_{M}\left(T-M_{f}\right)+b_{M} \sigma\right]$

Although there have many kinds of SDS-based actuators, this part only present the phase transformation modeling of the SDS-I as it is representative in these SDS-based actuators. For example, in the phase transformation model of the SDS-II actuator, it only increase an extra part in the stress factor of the SMA wires $\Lambda$ (detail in the Section S2 in the supplementary materials).

\subsection{Phase transformation simulation of the SDS-I}

Based on the equation (9), the simulation model of the SDS-I was built by using Matlab/Simulink software package (as shown in Figure S2). All the parameters were detail presented in the Table S3 in the supplementary materials. Figure 6 shows the bending responses of the SDS-I in the experiment and simulation. As a big heating current resulting a large inertia which cannot be simulated in the simulation, a current of 1 A was conducted on the SDS-I in the experiment. As a result, the SDS-I in the cooling process of the experiment presented an oscillation trend but slight degree. According to the Figure 6, in both start and end of the phase transformation, the experiment and simulation have gaps in the time responses: First, in the simulation, the SDS-I begun to bend at the time of $27 \mathrm{~ms}$ while the experiment one was at $5 \mathrm{~ms}$; 
second, at the $270 \mathrm{~ms}$ in the simulation, the SDS-I totally recovered to its original position while it presented the oscillation trend to gradually recover in the experiment. The above gaps were caused by the difference of the four phase transformation temperatures between the simulation and experiment. The four phase transformation temperatures respectively are austenite start and finish temperatures $\left(A_{s}, A_{f}\right)$, and martensitic start and finish temperatures $\left(M_{s}, M_{f}\right)$, which are four constant temperature values in the simulation. However, the four phase transformation temperatures only represent the main temperature intervals to occur the phase transformations. In practice, the SMA wires slightly but sure occur phase transformation before the temperatures $A_{s}$ and $M_{s}$ and after $A_{f}$ and $M_{f}$.

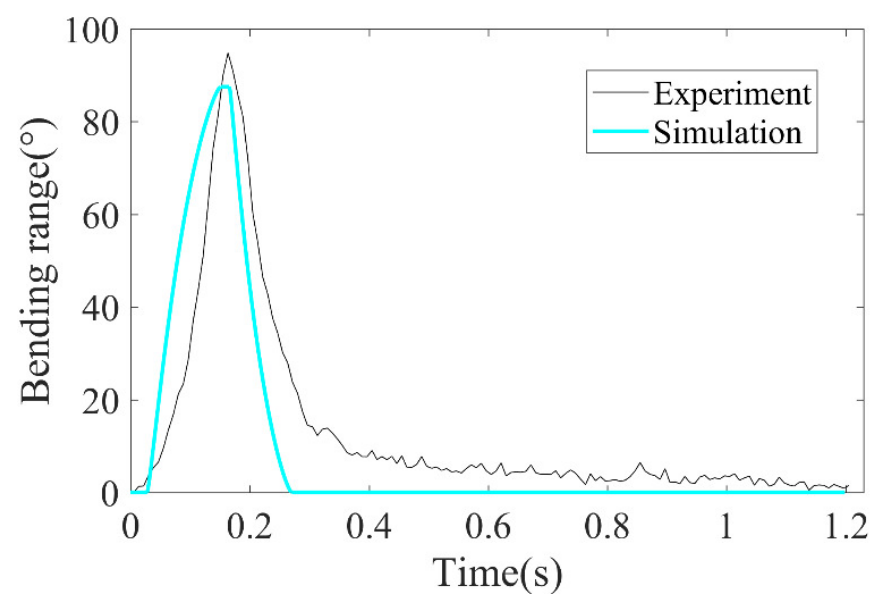

FiG. 6. Bending responses of the SDS-I in experiment and simulation.

Apart from the influence from phase transformation temperatures, the hypothesis (3) also resulted another mentionable gap between the experiment and simulation in their maximum bending states. The simulation has a short static state to cool the temperature of the SMA wires to its martensitic start temperature; however, in the experiment, the SDS-I immediately recoil to its original position without any static. Under the hypothesis (3), the SDS-I is a quasi-static state, and therefore it presented a static state in the simulation. Despite some differences, the simulation successfully tracked the bending response of the SDS-I. Besides, based on the simulation, it is facilitate to study the variations of the martensite fraction and strain of the SMA wires in the bending motion of SDS-I (detail in the Section S3 in the supplementary materials).

\section{Results}

\subsection{Soft Actuators SDS-I with Layered adhesive bonding}

Based on the layered adhesive bonding process, an actuator of SDS-I which is 91 $\mathrm{mm} \times 16 \mathrm{~mm} \times 2.5 \mathrm{~mm}$ and weighs $4.5 \mathrm{~g}$ was fabricated. The length of the SMA wires between two PCB boards is $80 \mathrm{~mm}$. After bonding, the SDS-I is flat, as shown in Figure 7a, but it cannot become flat again after being heated and bent to an angle of $17.5^{\circ}$ as shown in Figure 7b. An AR heating strategy [26] is used to regulate the heating process of SDS-I; here the heating times gradually decrease along with the 
heating current increases, as shown in the top left of Figure 7b. The AR heating strategy (more detail in the Section S4 in the supplementary materials) is a self-feedback system based on the resistance feedback of the SMA wires. Figure $4 \mathrm{~b}$ shows that the maximum bending ranges of SDS-I are $95^{\circ}, 114.5^{\circ}, 128.6^{\circ}$ and $135.7^{\circ}$ at a heating current of $1 \mathrm{~A}$ to $1.75 \mathrm{~A}$ with an interval of $0.25 \mathrm{~A}$. In this part, all the channels of the SDS-I were recruited. It can see that the maximum bending range of SDS-I increases as the heating current increases. This result has the same regulation with SMS, where the SMA wires are parallel with an elastic structure; it has a similar structure as SDS-I although they have different fabrication processes. Unlike the SMS, the SDS-I has a thinner, lighter structure, but has a larger bending range.

Besides, two other parameters of SDS-I were tested to reveal its capabilities. Figure $7 \mathrm{c}$ and $7 \mathrm{~d}$ show the response frequencies and frequency responses of SDS-I, respectively. The response frequency of SDS-I i.e., the response time in which SDS-I begins to bend and return to its position in a reciprocal motion where the heating process was regulated by the AR heating strategy. Figure $7 \mathrm{c}$ shows that the largest response frequency is $0.802 \mathrm{~Hz}$ at a heating current of $1 \mathrm{~A}$. This lower heating current will increase the heating time, which is faster in a higher heating current, but the stronger oscillation during the cooling process took longer time to become static.

Frequency responses are used to describe the dynamic property of a system. Under a reciprocal flapping motion, the temperature of SDS-I will increase and gradually stabilise at a temperature as the heat accumulates. In this process the bending range of SDS-I will decrease and gradually stabilise because the SMA wires cannot totally accomplishing the inverse martensitic phase transformation in the cooling process. To explore the frequency responses, a series of discrete frequencies in the SDS-I under reciprocal flapping motions were applied. We then measured the bending range of SDS-I when it is balanced. The bending range ratio indicates the balanced bending range divided by the maximum bending range (i.e. normalized by the maximum bending range of SDS-I). The result showed that the cut-off frequency of SDS-I is $1.178 \mathrm{~Hz}$, as shown in Figure 7d.
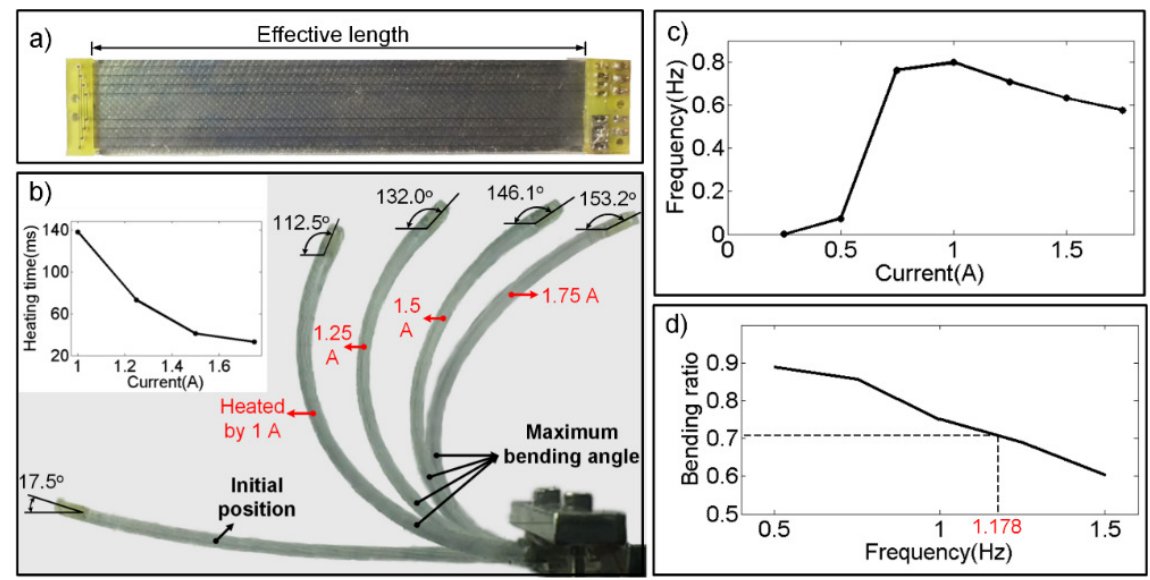

FIG. 7. Analysis of the SDS-I. a) Actuator SDS-I, b) the bending range of the SDS-I, c) and d) are the response frequencies and frequency responses of the SDS-I. 


\subsection{Soft Actuators SDS-II with Layered adhesive bonding}

The actuator SDS-II is capable of bi-directional planar motion because two SDSs are bonded to two sides of the spring steel. Figure 8a shows the bi-directional actuator of this $91 \mathrm{~mm} \times 16 \mathrm{~mm} \times 5 \mathrm{~mm}$ SDS-II which weighs $8 \mathrm{~g}$. Unlike SDS-I the initial position of SDS-II is almost vertical. So by actuating different channels, the bending angles of SDS-II are hierarchical and channelized. The biggest bending angles in channel $\mathrm{C} 1234$ of the right and left were $74.3^{\circ}$ and $73.0^{\circ}$, respectively. The totally bending range of SDS-II is $147.3^{\circ}$, which is bigger than SDS-I. Figure $8 \mathrm{~b}$ shows the bending ranges of SDS-II. It is easy to find a special property where the bending range of SDS-II between the right and left channel is consistent; the largest deviation is in the channel $\mathrm{C} 12$, with a $5 \%$ error between the bending ranges of the right and left channels. This consistency surely owed to the high degree of symmetry of SDS-II.

Figure $8 \mathrm{~b}$ shows that response of SDS-II is slow at a lower heating current and fast when the heating current is bigger. To study the force in different channels, a force sensor (made by Zhongnuo Incorporation, model: ZNLBM-5N) was used to measure the output force at the end of SDS-II. We carried out two experiments to measure the 'contact' force in at a low heating current of $0.5 \mathrm{~A}$, and the 'tap force' at heating current of a 1.25 A. The AR heating strategy is applied to regulate the heating process of SDS-II. Figure 8c shows the contact forces and tap forces of SDS-II in different channels; where the contact forces show a linear tendency, unlike the tap forces. The tap force is bigger than the contact force and both of them present a hierarchical output. This proves that the SDS-based actuator inherits the channelized and tunable properties of the SDS.
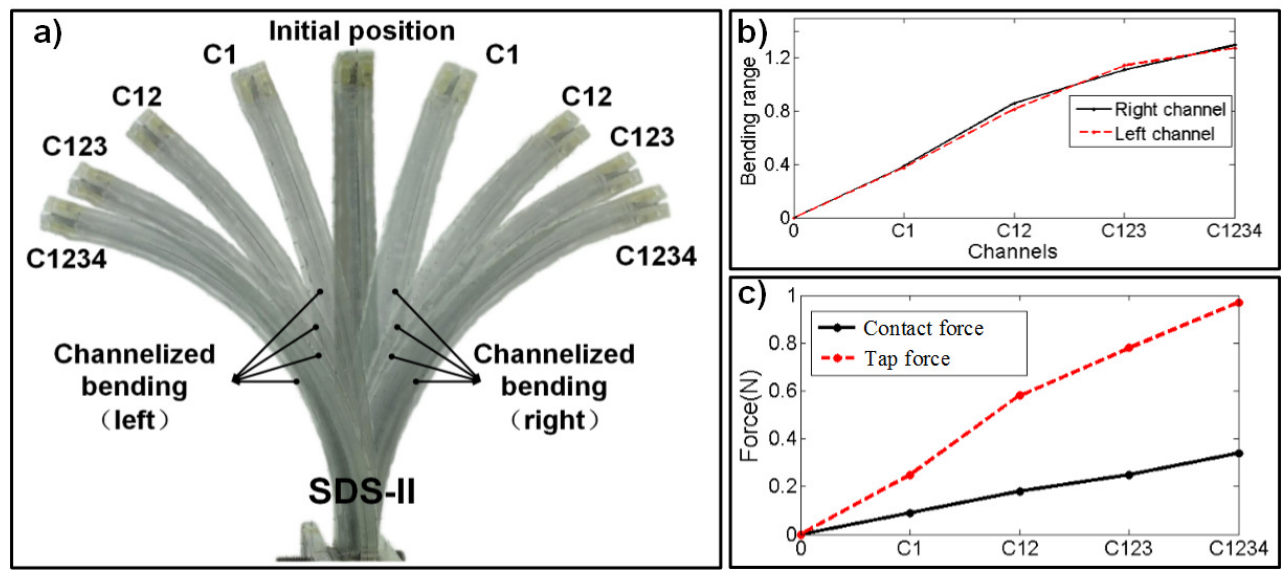

FIG. 8. Bi-directional actuator SDS-II. a) Bi-directional bending of SDS-II, b) bending ranges of SDS-II, c) contact force and tap force of SDS-II.

The actuator SDS-II is capable of symmetrical bi-directional motion due to its symmetrical structure where the two SDSs form the perfectly laminated plate. However, it is difficult to place the elastic plate in a symmetrical position using casting technology because the solution of soft materials cannot hold the elastic plate before solidification and it is difficult to add the support structures which hold the elastic plate into the actuator's molds, which only have limited space [31, 32]. In 
addition, a comparison experiment was conducted in the SDS-II to verify the steerability capabilities of the SDS-based actuators. First, we got the bending range of the SDS-II with all channels recruitment by using the adaptive heating strategy which has been proposed in the previous work [31]. As shown in Figure 9a, the bending range exhibited a small error when the resistance coefficient $\varepsilon=1$, however, had larger errors in the others. Such result is for the reason that the adaptive heating strategy only has a steady result when the $\varepsilon=1$ in which the phase transformation of the SMA wires has finished. Then, the bending ranges of the SDS-II with four different recruiting channels were obtained. All of them were regulated by the adaptive heating strategy, and their resistance coefficients $\varepsilon$ are 1. As shown in Figure 9b, although the bending ranges of $\mathrm{C} 1, \mathrm{C} 12$ and $\mathrm{C} 123$ respectively close to the ones in $\varepsilon=0.4,0.6$ and 0.8 , the former three have small errors. Based on the digital structures, the SDS-II achieves a reliable and tunable output.

Comparing to the work [30], the advantages of the layered adhesive bonding technology can be primary concluded as the following aspects. At first, the layered adhesive bonding technology simples the fabrication process and enables the SDS-based actuators with a light and compact body. Secondly, the soft actuators have a precise structure parameter, particular in the bi-directional actuator which is capable of a symmetrical motion. Besides, the digital design method also endows the SDS-based actuators with capabilities of tunable output and steerability.
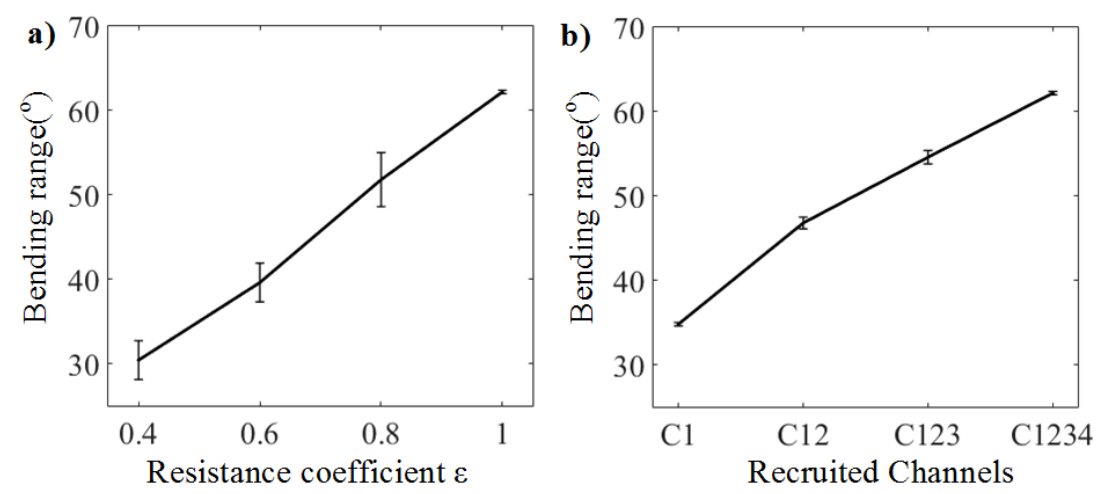

FiG. 9. Bending ranges of SDS-II under (a) different recruited channels and (b) different resistance coefficient of SMA wires.

\subsection{Extensive Soft Actuators with Layered adhesive bonding and Modular Assembly}

This part used SDS, SDS-I, and SDS-II as independent modular units to assemble multifarious soft actuators. We first made an SDS-based actuator by bonding three SDSs to a wide piece of spring steel based on the layered adhesive bonding process, as shown in Figure 10a. Although it has same function as SDS-I, the SDS-based actuator has a larger force output and a larger work space. It is easy to image that multifarious soft actuators can be designed by bonding SDSs with anolamous elastic structures. We then modularly assembled SDS-Is and SDS-IIs to make an actinormorphic soft actuator and an arm-like soft actuator, respectively. As Figure $10 \mathrm{~b}$ shows, the actinomorphic soft actuator was made by four SDS-Is with a central 
disc. Figure 10c shows the arm-like actuator by bonding two SDS-IIs.

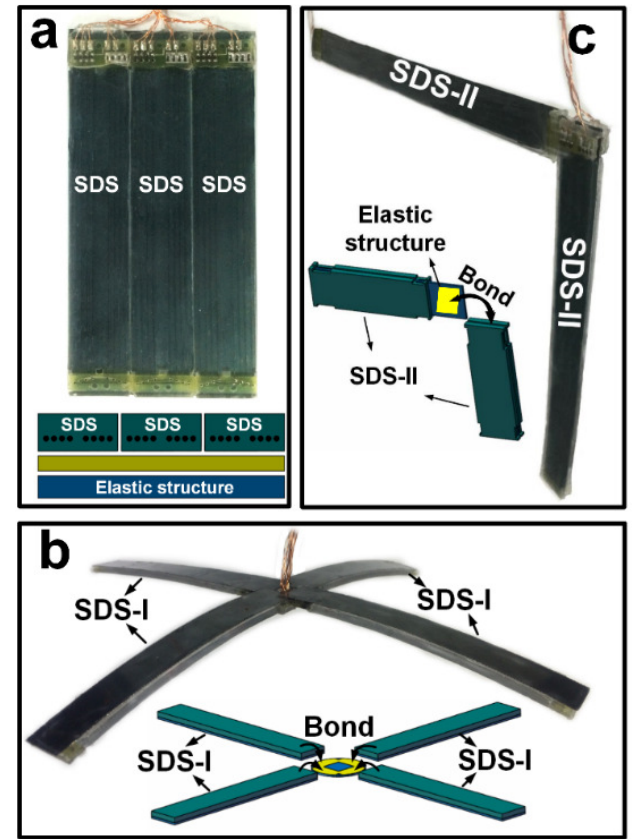

FIG. 10. Soft actuators by modularly assembling the SDSs, SDS-Is and SDS-IIs. a) SDS-based soft actuator by assembling the SDSs with an anomalous elastic structure,

b) SDS-I based soft actuator, c) SDS-II based soft actuator.

Owing to the modular assembly method, the diverse structures of the SDS-based soft actuator has different locomotion manners. The strategy of combining the modular assembly and layered adhesive bonding process helps to design soft actuators. The modular assembly is a quick and easy way to fabricate multifarious soft actuators, which will lower the cost, add diversity, and expand the application of SDS-based actuators.

\subsection{Tunable Output of Soft Actuators}

Using an actinomorphic soft actuator as an example, a series of jump tests were carried out to present a tunable output of SDS-based actuator, as shown in the Figure 11. The SDSs of the actinomorphic soft employed the SMA wires with a diameter of $0.15 \mathrm{~mm}$. The heating time in jumping motion was $20 \mathrm{~ms}$ at a heating current of $4 \mathrm{~A}$. As the heating time is short, the heating strategy in this part was open-loop. A high speed camera with a $5000 \mathrm{fps}$ was used to record the jumping motion. Figure 11a shows the jumping motion of the actinomorphic soft actuator with four channelized recruitment paradigm. In the tests, all the SDSs of the actinomorphic soft robot had a same recruited channel to achieve vertical jumps. As shown in Figure 11a, the actinomorphic soft actuator jumped a highest height of $157 \mathrm{~mm}$ which is more than 60 times of the actuator height at the channel of C1234, and at heights of $42 \mathrm{~mm}, 85 \mathrm{~mm}$, and $139 \mathrm{~mm}$ at the channels $\mathrm{C} 1, \mathrm{C} 12$, and $\mathrm{C} 123$, respectively. The jumping heights were measured by averaging the heights of the left and right SDS-Is. As shown in Figure 11b, the height ratios of four kinds of recruitments respectively are 1, 2.01, 3.31 and 3.73. Although they haven't a rigorous and equidifferent grade, the good 
performances of the error bars convince the soft actuator capable of tunable and hierarchical vertical jumping motion.

Furthermore, an experiment in which the robot arms were differential recruitment at the heating current of $4 \mathrm{~A}$ was conducted. As shown in Figure 12, two of arms were recruited in $\mathrm{C} 2$ and the other two were $\mathrm{C} 3$ and $\mathrm{C} 1$, respectively. Under such input channels, the actuator performed a directional jumping motion which directs to the $\mathrm{C} 1$ arm from the $\mathrm{C} 3$ arm. Besides, a zig-zag walking trend was presented in the directional jumping motion in which the average speed was approximately $46.6 \mathrm{~mm} / \mathrm{s}$ that is about 0.24 time of the body length of the actuator. The high speed camera with 1000 fps was used to record the directional jump of the actuator. Both the heights and directional displacement of actuator were obtained by using image processing on MATLAB platform.
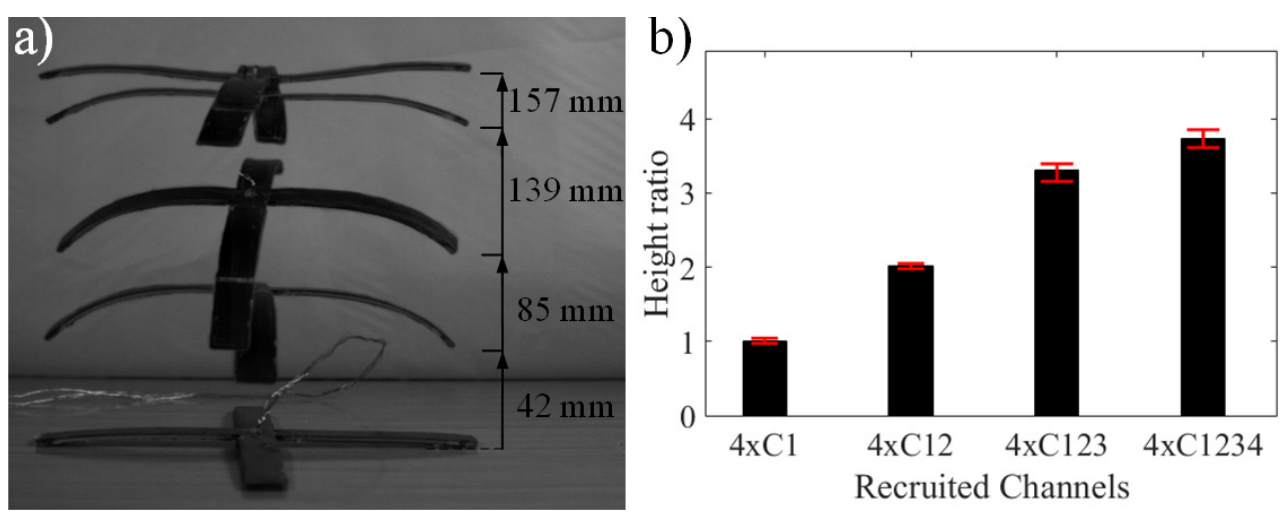

FIG. 11. The tunable vertical jump of the actinomorphic soft actuator. a) The jumping motions at four recruited channels and $b$ ) its height ratio.

The above results show that the actinomorphic soft actuator was capable of a jumping motion based on the impulsive response of the SMA wires. Most importantly, the actuator performed tunable and steerable jumps in vertical and directional jumping motions by digital recruiting the SDSs. Obviously, the tunable output capability enables the actinomorphic soft actuator channelises its movement, and therefore being steerable than the current soft jumping robots actuated by combustion $[2,4,40]$ and explosion [3]. 

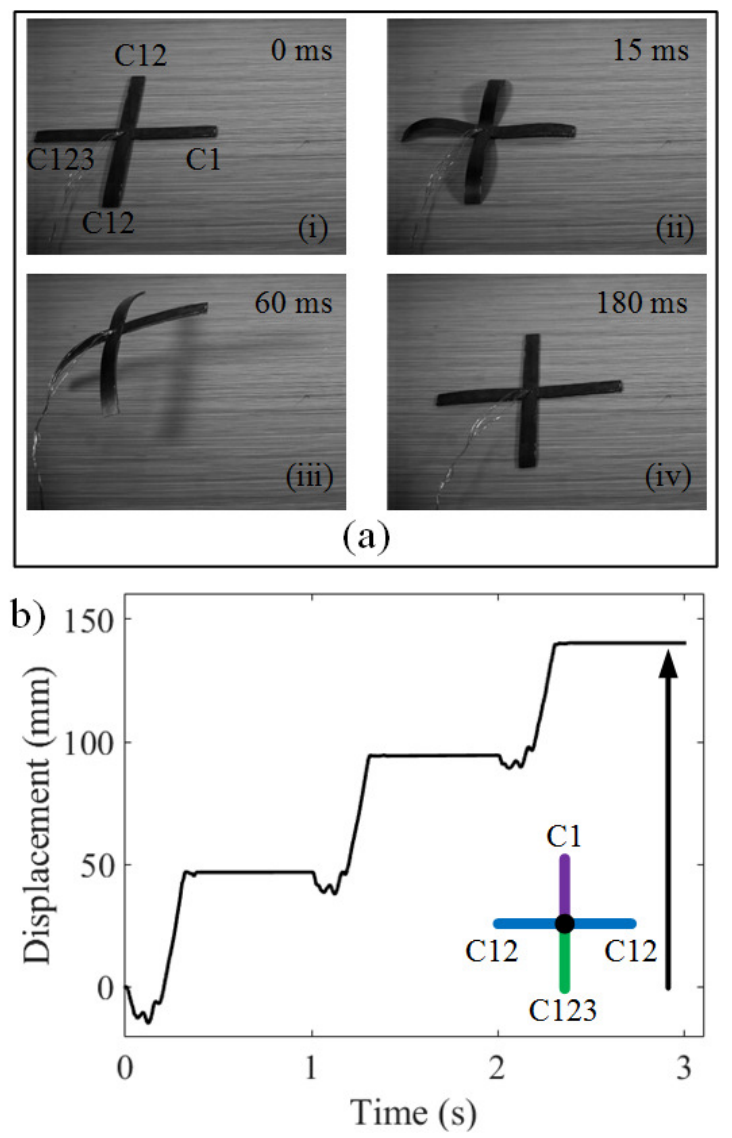

FIG. 12. Tunable directional jump of the actinomorphic soft actuator. a) The tunable directional jumping motion and $b$ ) its displacement.

\section{Conclusion}

This article proposed a novel SMA-based structure called SDS to assemble soft actuators based on a layered adhesive bonding technology. There are four independent, parallel, and coplanar SMA wires arranging in the structure, which endows the SDS with digital output. A series of tests were carried out to measure the output forces of SDS, which presented a hierarchy and channelised output, thus providing a tunable method. The layered adhesive bonding technology was used to bond the SDSs with an elastic backbone to fabricate SDS-based actuators, e.g., actuators SDS-I and SDS-II by bonding the spring steel with an SDS and two SDSs, respectively. The response frequencies and frequency responses of SDS-I were obtained based on experimental tests, and the best response frequency was $1 \mathrm{~Hz}$ and the cut-off frequency was 1.178 Hz. With two SDSs symmetrically bonded to spring steel, SDS-II is capable of outputting a bi-directional motion with good symmetry and consistency, and also showed a channelised and tunable force outputs at the same time. The digital design method endows the SDS-based actuators with capabilities of tunable output and steerability.

The phase transformation dynamic model of the SDS-I was built, and a comparison was conducted between bending responses in the experiment and simulation. The thermodynamics of the SDS-I actuator was conducted to analyse its 
temperature field and heat accumulation phenomenon. By taking SDS, SDS-I and SDS-II as independent units, the modular assembly method was used to fabricate different soft actuators. There are two typical assembly processes: one is modularly assembling a number of SDSs with an anomalous elastic plate; the other is modularly assembling SDS-Is and SDS-IIs. Modular assembling endows the fabricated soft actuators with diverse capabilities and multiple motion degrees. Owing to the digital structure, the layered adhesive bonding process and modular assembly, the actinomorphic soft actuator achieved channelised, tunable and steerable jumps in vertical and directional motions, and reached a height of $157 \mathrm{~mm}$ and an average of $46 \mathrm{~mm} / \mathrm{s}$, respectively.

In the future works, structure optimization of the SDS and promotion of the manufacturing processes will be conducted to improve the equidifferent degree of the tunable output of the SDS-based actuators. Based on the SDS and modular assembly, it will facilitate to design and fabricate bionic soft robots with symmetrical bodies, e.g., starfish-like, jellyfish-like and fish-like robots. Besides, some state of the art control algorithms, e.g., PID controller, will be incorporated with the self-feedback control strategy to further improve the intellectuality of the SDS.

\section{Acknowledgement}

This work was supported from the National Natural Science Foundation of China (Nos. 61773358, 51705495 and 51105349).

\section{Author Disclosure Statement}

The authors declare that no competing financial interests exist.

\section{References}

[1] Fratzl P, Barth F G. Biomaterial systems for mechanosensing and actuation[J]. Nature, 2009 November; 462(7272): 442-448.

[2] Loepfe M, Schumacher C M, Lustenberger U B, et al. An untethered, jumping roly-poly soft robot driven by combustion[J]. Soft Robot, 2015, 2(1): 33-41.

[3] Shepherd R F, Stokes A A, Freake J, Barber J, Snyder P W. Using explosions to power a soft robot[J]. Angew Chem Int Edit, 2013 March, 52(10): 2892-2896.

[4] Bartlett N W, Tolley M T, Overvelde J T B, Weaver J C, Mosadegh B. A 3D-printed, functionally graded soft robot powered by combustion[J]. Science, 2015 July; 349(6244): 161-165.

[5] Kim S, Laschi C, Trimmer B. Soft robotics: a bioinspired evolution in robotics[J]. Trends Biotechnol, 2013 April; 31(5): 287-294.

[6] Pfeifer R, Lungarella M, Iida F. Self-organization, embodiment, and biologically inspired robotics[J]. Science, 2007 November; 318(5853): 1088-1093. 
[7] Rus D, Tolley M T. Design, fabrication and control of soft robots[J]. Nature, 2015 May; 521(7553): 467-475.

[8] Kovač M. The bioinspiration design paradigm: A perspective for soft robotics[J]. Soft Robot, 2014 March; 1(1): 28-37.

[9] Laschi C, Cianchetti M, Mazzolai B, Margheri L, Follador M, Dario P. Soft robot arm inspired by the octopus[J]. Adv Robotics, 2012 January, 26(7): 709-727.

[10] Manti M, Hassan T, Passetti G, d'Elia N, Cianchetti M, Laschi C. A bioinspired soft robotic gripper for adaptable and effective grasping [J]. Soft Robot, 2015 August, 2(3): 107-116.

[11] Rodrigue, H., Wang, W., Han, M. M., Quan, Y. J., Ahn, S. H. Comparison of mold designs for SMA-based twisting soft actuator[J]. Sensor Actuat A-Phys, 2015 November; 237: 96-106.

[12] Villoslada, A., Flores, A., Copaci, D., Blanco, D., Moreno, L. High-displacement flexible Shape Memory Alloy actuator for soft wearable robots[J]. Robot Auton Syst, 2015 January; 73: 91-101.

[13] Jin H, Dong E, Mao S, Xu M, Yang J. Locomotion modeling of an actinomorphic soft robot actuated by SMA springs[C]//Robotics and Biomimetics (ROBIO), 2014 IEEE International Conference on. IEEE, 2014, pp. 21-26.

[14] Mao S, Dong E, Jin H, Xu M, Zhang S, Yang J, Low K H. Gait Study and Pattern Generation of a Starfish-Like Soft Robot with Flexible Rays Actuated by SMAs[J]. J Bionic Eng, 2014 July; 11(3): 400-411.

[15] Brinson L C, Bekker A, Hwang S. Deformation of shape memory alloys due to thermo-induced transformation[J]. J Intel Mat Syst Str, 1996 January; 7(1): 97-107.

[16] Lagoudas D C, Bo Z, Qidwai M A. A unified thermodynamic constitutive model for SMA and finite element analysis of active metal matrix composites[J]. Mech Compos Mater St, 1996 February; 3(2): 153-179.

[17] Zhu S, Zhang Y. A thermomechanical constitutive model for superelastic SMA wire with strain-rate dependence[J]. Smart Mater Struct, 2007 August; 16(5): 1696.

[18] Ma N, Song G. Control of shape memory alloy actuator using pulse width modulation[J]. Smart Mater Struct, 2003 September; 12(5): 712.

[19] Teh Y H, Featherstone R. An architecture for fast and accurate control of shape memory alloy actuators[J]. Int J Robot Res, 2008 May; 27(5): 595-611.

[20] Gurung H, Banerjee A. Development of an extended Kalman filter for the self-sensing application of a spring-biased shape memory alloy wire actuator[J]. Smart Mater Struct, 2016 February; 25(2): 025012.

[21] Guo Z, Pan Y, Wee L B, Wee L B, Yu H. Design and control of a novel compliant differential shape memory alloy actuator[J]. Sensor Actuat A-Phys, 2015 April; 225: 71-80.

[22] Furst S J, Seelecke S. Modeling and experimental characterization of the stress, strain, and resistance of shape memory alloy actuator wires with controlled power input[J]. J Intel Mat Syst 
Str, 2012 June; 23(11): 1233-1247.

[23] Jin, H., Dong, E., Alici, G., Mao, S., Min, X., Liu, C., K H Low, \& Yang, J. A starfish robot based on soft and smart modular structure (SMS) actuated by SMA wires. Bioinspiration \& biomimetics, 2016, 11(5), 056012.

[24] Ma N, Song G, Lee H J. Position control of shape memory alloy actuators with internal electrical resistance feedback using neural networks[J]. Smart Mater Struct, 2004 August; 13(4): 777.

[25] Wang, T. M., Shi, Z. Y., Liu, D., Ma, C., \& Zhang, Z. H. An accurately controlled antagonistic shape memory alloy actuator with self-sensing[J]. Sensors-Basel, 2012 June; 12(6): 7682-7700.

[26] Kannan S, Giraud-Audine C, Patoor E. Application of Laguerre based adaptive predictive control to shape memory alloy (SMA) actuator[J]. Isa T, 2013 March; 52(4): 469-479.

[27] Kannan S, Giraud-Audine C, Patoor E. Laguerre model based adaptive control of antagonistic shape memory alloy (SMA) actuator[C]/SPIE Smart Structures and Materials+ Nondestructive Evaluation and Health Monitoring. International Society for Optics and Photonics, 2010, pp. 764307-764307-12.

[28] Barrett, R., \& Gross, R. S. Super-active shape-memory alloy composites[J]. Smart Mater Struct, 1996 June, 5(3), 255-260.

[29] Rodrigue H, Wang W, Bhandari B, Han M W, Ahn S H. SMA-based smart soft composite structure capable of multiple modes of actuation[J]. Compos Part B-Eng, 2015 Decemeber; 82: 152-158.

[30] Rodrigue H, Wei W, Bhandari B, Ahn S H. Fabrication of wrist-like SMA-based actuator by double smart soft composite casting[J]. Smart Mater Struct, 2015 December; 24(12): 125003.

[31] Jin H, Dong E, Xu M, et al. Soft and smart modular structures actuated by shape memory alloy (SMA) wires as tentacles of soft robots[J]. Smart Mater Struct, 2016 July; 25(8): 085026.

[32] Braun S, Sandstrom N, Stemme G, Van D. Wafer-scale manufacturing of bulk shape-memory-alloy microactuators based on adhesive bonding of titanium-nickel sheets to structured silicon wafers[J]. J Microelectromech S J, 2009 December, 18(6): 1309-1317.

[33] Gradin H, Braun S, Stemme G, Wijngaart W. SMA microvalves for very large gas flow control manufactured using wafer-level eutectic bonding[J]. IEEE T Ind Electron, 2012 December; 59(12): 4895-4906.

[34] Gradin H, Bushra S, Braun S, Stemme G, Wijngaart W. Wafer-level integration of NiTi shape memory alloy on silicon using Au-Si eutectic bonding[J]. J Micromech Microeng, 2012 November; 23(1): 015008.

[35] Ali M S M, Takahata K. Frequency-controlled wireless shape-memory-alloy microactuators integrated using an electroplating bonding process[J]. Sensor Actuat A-Phys, 2010 August; 163(1): 363-372. 
[36] Zhang J J, Yin Y H, Zhu J Y. Electrical resistivity-based study of self-sensing properties for shape memory alloy-actuated artificial muscle[J]. Sensors-Basel, 2013 September; 13(10): 12958-12974.

[37] Martinez R V, Glavan A C, Keplinger C, Oyetibo A I, Whitesides G M. Soft actuators and robots that are resistant to mechanical damage[J]. Adv Funct Mater, 2014 February; 24(20): 3003-3010.

[38] Ahmadzadeh H, Masehian E, Asadpour M. Modular Robotic Systems: Characteristics and Applications[J]. J Intell Robot Syst, 2015 June; 81(3-4): 317-357.

[39] Kwok S W, Morin S A, Mosadegh B, So J, Shepherd R F, Martinez R V, Smith B, Simeone F C, Stokes A A, Whitesides G M. Magnetic assembly of soft robots with hard components[J]. Adv Funct Mater, 2013 December, 24(15): 2180-2187.

[40] Tolley M T, Shepherd R F, Karpelson M, Bartlett N W, Galloway K C. An untethered jumping soft robot[C]//2014 IEEE/RSJ International Conference on Intelligent Robots and Systems. IEEE, 2014, pp. 561-566. 


\section{Supplementary materials}

\section{Tunable Smart Digital Structure (SDS) to Modularly Assemble Soft Actuators with Layered Adhesive Bonding}

By Hu Jin, Erbao Dong, Min Xu, Qirong Xia, Shuai Liu, Weihua Li, Jie Yang

\section{Material properties and boundary condition in the COMSOL simulation}

The heat transfer module was applied in the COMSOL simulation. The simulation used the solid heat transfer part which is mainly based on the following heat balance equation:

$$
\rho C_{p} \frac{\partial T}{\partial t}+\rho C_{p} \mathbf{u} \cdot \nabla T+\nabla \cdot(-k \nabla T)=\mathrm{Q}
$$

where $\rho$ is the density of the objects, $k$ represents the thermal conductivity, $C_{p}$ is the specific heat capacity, and $Q$ is the heating power per unit volume equal to 967.7 $\mathrm{W} / \mathrm{m}^{3}$. $T$ represents the temperature of the model. The simulation dimension and material properties are shown in Table S1 and Table S2, respectively. It's worth mentioned that the materials were selected from the material libraries of COMSOL Multiphysics. Some properties of the materials are defined by the software and most of these properties change with the temperature of the material.

Table S1. The dimensions of the simulation model.

\begin{tabular}{c|c|c}
\hline \multicolumn{2}{c|}{} & Dimension \\
\hline \multirow{3}{*}{ Simulation model } & Length $(\mathrm{mm})$ & 80 \\
\cline { 2 - 3 } & Width $(\mathrm{mm})$ & 16 \\
\cline { 2 - 3 } & Thickness $(\mathrm{mm})$ & 2.5 \\
\hline \multirow{2}{*}{ The Spring steel } & Length $(\mathrm{mm})$ & 80 \\
\cline { 2 - 3 } & Width $(\mathrm{mm})$ & 16 \\
\cline { 2 - 3 } & Thickness $(\mathrm{mm})$ & 0.1 \\
\hline \multicolumn{2}{c}{ Diameter of the SMA wires (mm) } & 0.15 \\
\hline \multicolumn{2}{c}{ Adjacent distances of the SMA wires (mm) } & 8 \\
\hline \multicolumn{2}{c}{ Distance between the SMA wires and the PVC } & 0.9 \\
\hline
\end{tabular}


Table S2. Material properties in the simulation model.

\begin{tabular}{cccc}
\hline $\begin{array}{c}\text { Material } \\
\text { property }\end{array}$ & $\begin{array}{c}\text { Heat source } \\
\text { (SMA) }\end{array}$ & PDMS & $\begin{array}{c}\text { Spring } \\
\text { steel }\end{array}$ \\
\hline$\rho\left[\mathrm{kg} / \mathrm{m}^{3}\right]$ & 6450 & 970 & $7.85 \times 10^{6}$ \\
\hline$C_{p}[\mathrm{~J} /(\mathrm{kg} \cdot \mathrm{K})]$ & 837 & 1460 & 460 \\
\hline$k[\mathrm{~W} /(\mathrm{m} \cdot \mathrm{K})]$ & 18 & 0.16 & 22 \\
\hline
\end{tabular}

The model applied continuity of temperature and heat flux at all interior boundaries. The heat flux boundary conditions can be described by the following equation:

$$
\mathbf{n} \cdot(k \nabla T)=h_{\mathrm{a}}\left(T_{\text {ext }}-T\right)
$$

where $\mathbf{n}$ represents the normal vector of the boundary, $T_{\text {ext }}$ is the external temperature (room temperature) and $h_{a}$ is the air heat transfer coefficient. Generally, in the case of the air natural convection, the heat transfer coefficient is $5 \sim 25 \mathrm{~W} /\left(\mathrm{m}^{2} \cdot \mathrm{K}\right)$. In this model, the air heat transfer coefficient is set to $15 \mathrm{~W} /\left(\mathrm{m}^{2} \cdot \mathrm{K}\right)$.

\section{Phase transformation dynamics of SDS-II actuator}

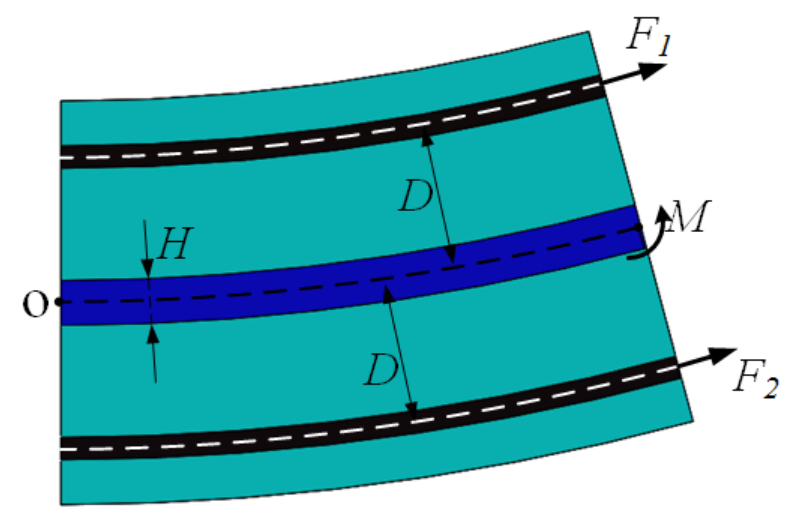

FIG. S1. Schematics of the SDS-II actuator

The bi-directional soft actuator SDS-II has two sets of SMA wires symmetrically distributing by the elastic plate. When heating one set of SMA wires, the other set of SMA wires will be extended, as shown in Figure S1. Therefore,

$$
\varepsilon_{2}=2 \varepsilon_{0}-\varepsilon_{1}
$$


$\varepsilon_{1}$ and $\varepsilon_{2}$ respectively are the stresses of the 1 th and 2nd SMA wires. The resultant moment is 0 :

$$
F_{1} D-F_{2} D=M_{\mathrm{e}}
$$

where $F_{1}$ and $F_{2}$ respectively are the forces of the 1th and 2nd SMA wires, and $F_{2}=E_{M} A\left(\varepsilon_{2}-\varepsilon_{0}\right)=E_{M} A\left(\varepsilon_{0}-\varepsilon_{1}\right) . E_{M}$ is the Young's modulus of the SMA wires in the martensite phase. Similarly, we can derive the phase transformation model of the SDS-II as shown in following:

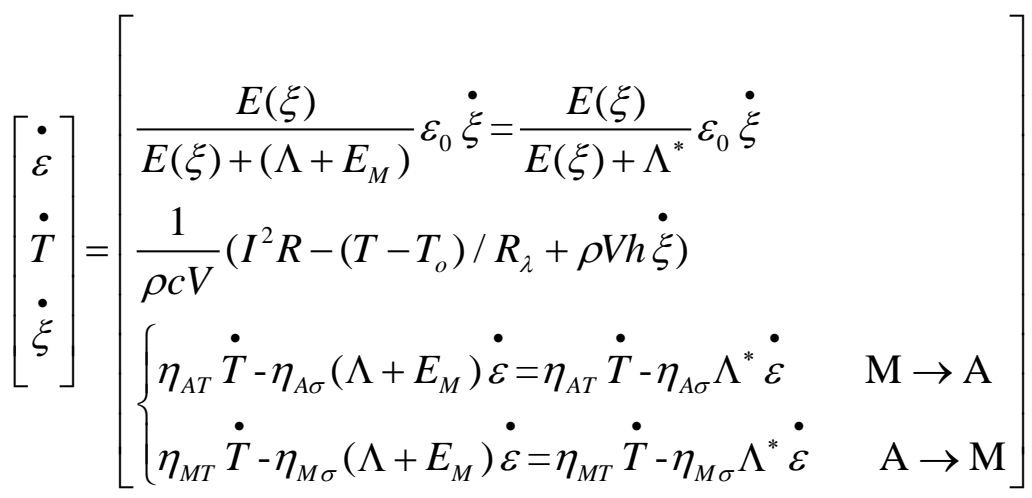

Where $\Lambda^{*}=\Lambda+E_{M}$. Apparently, the phase transformation model of the SDS-II has a same pattern with the SDS-I: they only have a difference in the stress factor of the SMA wires.

3. Phase transformation dynamic simulation of SDS-I based on Matlab platform

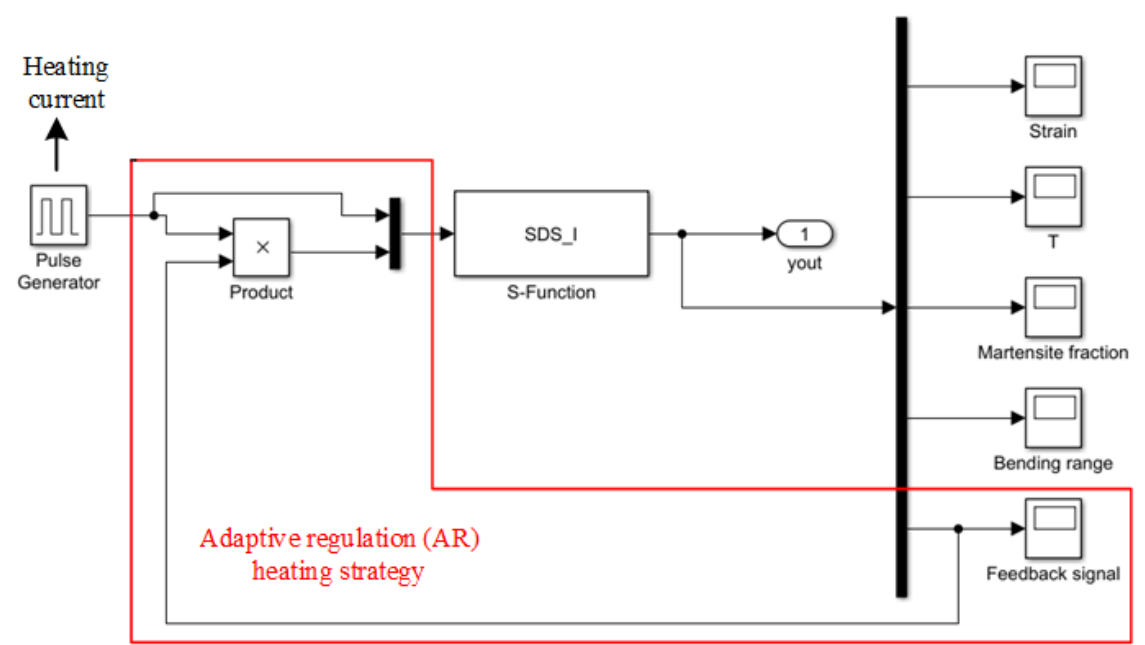


FiG. S2. Simulink project of the SDS-I

Figure S2 shows the Simulink project of the SDS-I based on the Matlab/Simulink software package. In the simulation, the adaptive regulation heating strategy was applied, as shown the red frame, to make the simulation have a same heating method with the experiment. Figure S3 and S4 respectively show the strain and martensite fraction of the SMA wires in the simulation.

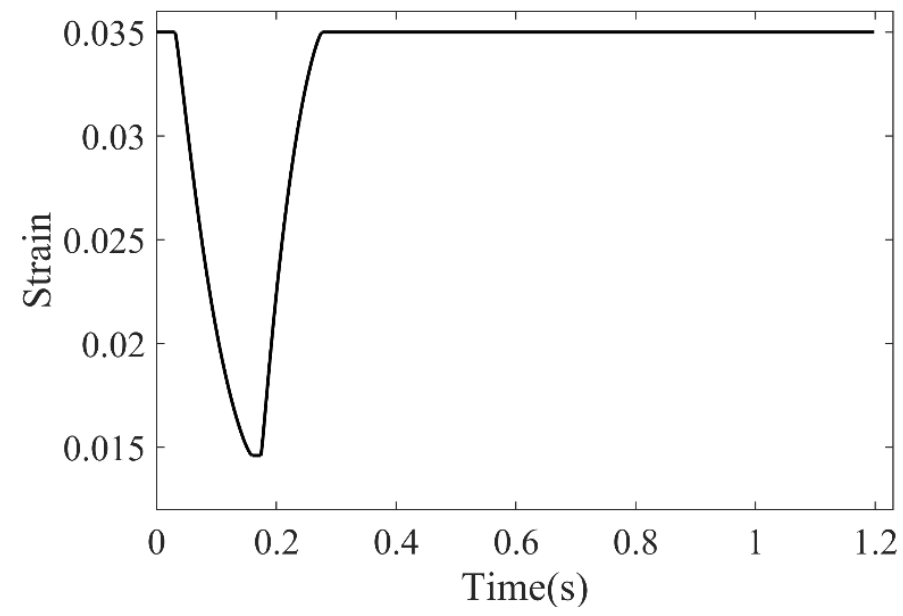

FiG. S3. The strain of the SMA wires in the simulation.

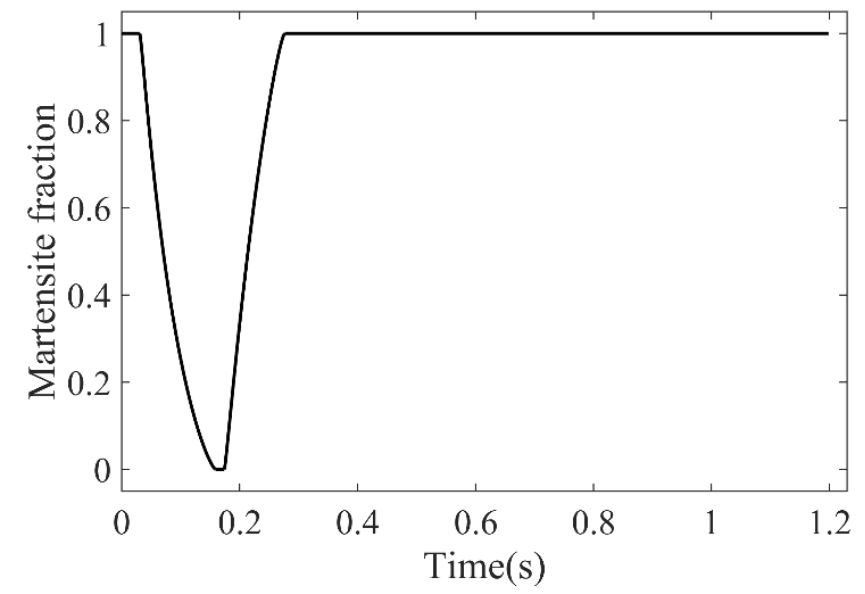

FiG. S4. The martensite fraction of the SMA wires in the simulation.

Table S3. Material properties in the simulation.

\begin{tabular}{|c|c|}
\hline Description (parameter) & Value (unit) \\
\hline Elastic modulus of spring steel $(E e)$ & $200 \mathrm{GPa}$ \\
\hline Size of the spring steel (width $(H) * \operatorname{high}(b))$ & $0.1 \times 16 \mathrm{~mm}$ \\
\hline
\end{tabular}




\begin{tabular}{|c|c|}
\hline Distance between the SMA wires and the spring steel $(D)$ & $1 \mathrm{~mm}$ \\
\hline Diameter of the SMA wires $(d)$ & $0.1 \mathrm{~mm}$ \\
\hline Length of the SMA wires & $80 \mathrm{~mm}$ \\
\hline Resistance per length of the SMA wires & $120(\Omega / \mathrm{mm})$ \\
\hline Density of the SMA wires $(\rho)$ & $6.45 \mathrm{~g} / \mathrm{cm}^{3}$ \\
\hline Specific heat capacity $(c)$ & $837 \mathrm{~J}\left(\mathrm{~kg} \cdot{ }^{\circ} \mathrm{C}\right)$ \\
\hline Latent heat of transformation of the SMA wires $(h)$ & $24.2 \times 10^{3} \mathrm{~J} / \mathrm{kg}$ \\
\hline Initial strain of the SMA wires $\left(\varepsilon_{0}\right)$ & 0.035 \\
\hline Austenite start temperature $\left(A_{s}\right)$ & $80^{\circ} \mathrm{C}$ \\
\hline Austenite finish temperature $\left(A_{f}\right)$ & $108{ }^{\circ} \mathrm{C}$ \\
\hline Martensitic start temperature $\left(M_{s}\right)$ & $78^{\circ} \mathrm{C}$ \\
\hline Martensitic finish temperature $\left(M_{f}\right)$ & $60^{\circ} \mathrm{C}$ \\
\hline Environment temperature $\left(T_{o}\right)$ & $15^{\circ} \mathrm{C}$ \\
\hline Effect of stress on austenite temperature $\left(C_{A}\right)$ & $15 \mathrm{MPa} /{ }^{\circ} \mathrm{C}$ \\
\hline Effect of stress on Martensitic temperature $\left(C_{M}\right)$ & $15 \mathrm{MPa} /{ }^{\circ} \mathrm{C}$ \\
\hline Initial martensite fraction $\left(\xi_{M}\right)$ & 0.99 \\
\hline Martensite fraction in Austenite finishing $\left(\xi_{A}\right)$ & 0.01 \\
\hline Austenitic Young's modulus $\left(E_{A}\right)$ & 78 GPa \\
\hline Martensitic Young's modulus $\left(E_{M}\right)$ & $23 \mathrm{GPa}$ \\
\hline coefficient for the thermal conductivity of the PDMS $(\lambda)$ & $0.280 \mathrm{~W} /(\mathrm{m} \cdot \mathrm{K})$ \\
\hline Equivalent diameter of PDMS $\left(d_{p d m s}\right)$ & $1 \mathrm{~mm}$ \\
\hline
\end{tabular}

\section{Adaptive regulation (AR) heating strategy}

SMAs refer to a group of alloys that possess the properties of shape memory effect (SME) and pseudoelasticity. They have different shape, mechanical and electrical properties in different phases. As a consequence, a variation in their electrical properties leads to a fluctuation in their resistance, which is stable when the phase transformation is completed. The resistance ratio is the instantaneous resistance of the SMA wires divided by their initial resistance at room temperature, where the 
SMA wires do not go through a phase transformation. Figure S5a displays five sets of the same resistance ratios of the SMA wires, and point A and point B respectively represent the starting and finishing times of the phase transformation process. As shown in Figure S5a, the resistances of the SMA wires have two extreme values among its phase transformation process, and the two extreme values respectively close to their phase transformation starting (point A) and finishing (point B) time. By obtaining the minimum resistance of the SMA wires (point B), the SMA-based actuator can adaptively regulate its heating process without overheating phenomenon. In our article, such control strategy was named as adaptive regulation (AR) heating strategy.
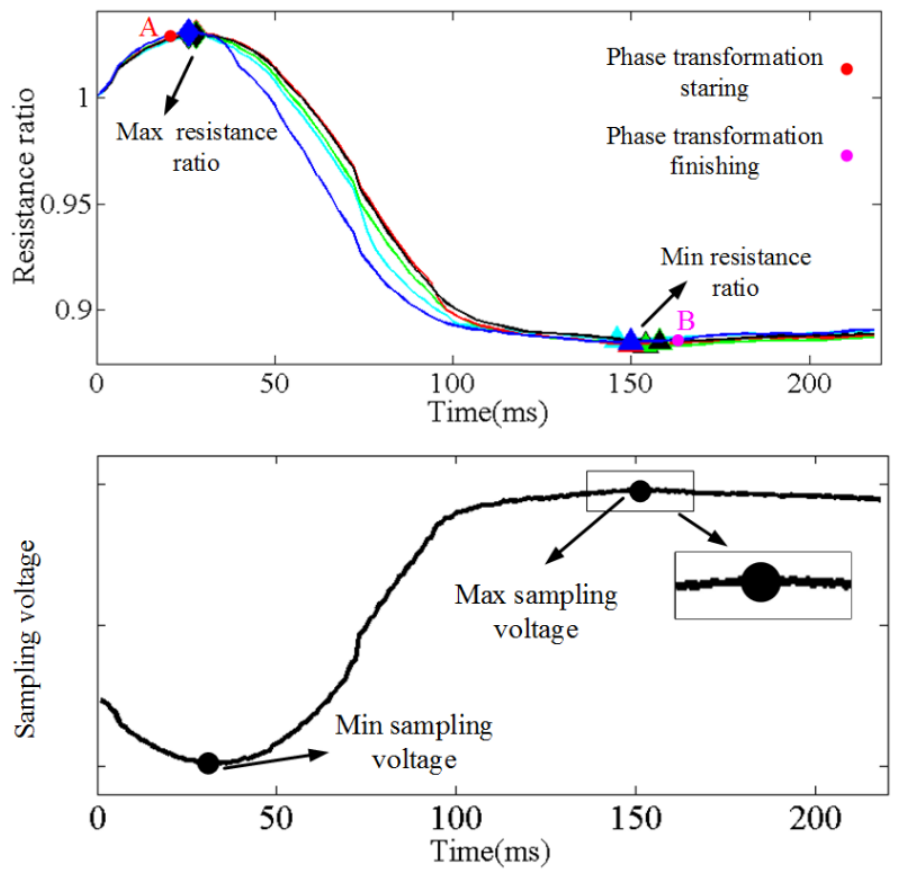

FIG. S5. The AR heating strategy for the SMA-based actuators. a) The resistance ratio of the heating process for the SMA wires. b) The sampling voltage across the constant resistor.

In the control circuit of the AR heating strategy, we applied a constant voltage as the input. A constant resistor was connected with the SMS in series to detect the resistance variation by sampling the voltage across the constant resistor. As shown in Figure S5b, the voltage across the constant resistor shows an inverse relation with the resistance ratio of the SMA wires. This follows that the resistance ratio variation is identified by simply sampling the voltage across the constant resistor. 
